\title{
Chapter 2 \\ The Joint Economic Impact Level (jEIL): A Decision Metric for Integrated Pest and Pollinator Management
}

\author{
Adam Flöhr, Johan A. Stenberg, and Paul A. Egan
}

\subsection{Introduction}

Injury from pests (including pest insects, weeds, and pathogens) and insect-provided pollination services are of large relevance to yield in the majority of the world's food crops (Klein et al. 2007; Oerke 2006). Various strategies are employed to manage these processes. For pests, Integrated Pest Management (IPM) incorporates a variety of cultural, mechanical, and biological control practices designed to supress pest populations in ways that are complementary, and which minimize the use of chemical pesticides (Stenberg 2017). For pollinators, managed bees are often used to boost crop pollination; in addition to agro-ecological practices (such as perennial flower strip plantings and the designation of no-till zones) designed to provision wild pollinators with forage and nesting resources (Garibaldi et al. 2017; Isaacs et al. 2017).

The effects of practices employed to manage pests and pollinators are not necessarily independent, however. Recent years have witnessed growing awareness of the widespread acute and chronic effects of pesticides on pollinators such as wild and managed bees (Godfray et al. 2014; Siviter et al. 2018). Furthermore, these effects can translate into impacts on crop pollination service (Brittain and Potts 2011; Stanley et al. 2015). Hence, owing to the potentially large and negative effects of pesticides on pollinators, up-take of more balanced pest control strategies - such as those inherent in an IPM approach - are increasingly demanded (Dicks

\footnotetext{
A. Flöhr

Department of Biosystems and Technology, Swedish University of Agricultural Sciences, Alnarp, Sweden

J. A. Stenberg · P. A. Egan $(\bowtie)$

Department of Plant Protection Biology, Swedish University of Agricultural Sciences, Alnarp,

Sweden

e-mail: paul.egan@slu.se
} 
et al. 2016; Bartomeus and Dicks 2018). This explicit incorporation into IPM of the goal of protecting pollinators from the harmful effects of pesticides has been termed by Biddinger and Rajotte (2015) as Integrated Pest and Pollinator Management (IPPM), and was developed into an expanded framework by Egan et al. (2020).

Here, we introduce a decision metric for IPPM termed the joint Economic Impact Level (jEIL). Akin to the Economic Injury Level (EIL - Stern et al. 1959; Pedigo and Rice 2014) which underpins IPM decision making, the joint EIL can be used to weigh the economic rationale for pest and pollinator management in a coordinated manner. This metric is not biased towards either practice however, and its use is equally valid where one or both are of primary concern. In particular, the joint EIL can account for how pest and pollinator management practices may interact, such as where the implementation of one can influence the need for the other. For instance, is the benefit of pest control economically justified when a practice (e.g. insecticide use; eradication of flowering weeds) also results in an additional cost to enhance pollination service? Given the current field density of pests and pollinators in a crop, and their specific relation to yield, which management practice (if any) should be prioritised? The joint EIL can be used to tackle such considerations and scenarios, in providing a foundational basis for decision making in IPPM.

Conceptual development of the joint EIL requires substantial formulaic elaboration of the EIL, as documented in the following sections. The EIL traditionally defines the point (in pest population density units) at which a control intervention becomes economically justified - i.e. the point where economic benefit outweighs cost. Hence, action is usually taken once, and only if, pest levels pass an Action Threshold (AT - set for instance at $80 \%$ EIL) in order to prevent the EIL being reached. Although not without their drawbacks, pest EILs have successfully underpinned IPM decision making for decades (Pedigo et al. 1986). We posit that expanding this metric to support pollinator management - and, by extension, a more formalised decision making framework for IPPM - could similarly prove of large benefit.

Development of the joint EIL also requires harmonization of the diverse sets of terminology typical of the pest and pollinator literature. In contrast to pest control, the goal of pollinator management is somewhat inverted; in that it is primarily motivated towards avoidance of yield limitation (as opposed to yield loss), and the maintenance of pollinator populations above (as opposed to below) a certain threshold. Below an optimal density (or visitation rate) of pollinators, pollination deficits and impacts on yield quantity and quality become increasingly apparent in pollinator-dependant crops. Such thresholds are highly crop specific, however, as governed by the large gradient in pollinator reliance evident across crops (Klein et al. 2007). Hence, in respect of the differing yield influences of pests and pollinators, and their associated management goals, we here apply the more general term impact (within joint Economic Impact Level) to reflect its wider incorporation of yield injury and limitation.

In the following sections, we take a step-wise approach to documenting the rationale behind how the joint EIL is constructed and can be used. We start by 
recounting construction of the traditional EIL and introducing here a pollinator equivalent (PEIL - the Pollinator Economic Impact Level), before describing the means through which these univariate cases are incorporated within the joint EIL. We follow these theoretical sections with a practical worked example for strawberry, using empirical data to calculate a joint EIL in aid of management decision making. Finally, we discuss broader issues in relation to the joint EIL, including recommendations for its practical use, and possible future developments.

\subsection{Derivation of EIL and Pollinator EIL}

\subsubsection{Pest Economic Injury Level with Constant Cost}

We begin by deriving the classic EIL for pests. The basis of the EIL is a simple economic model in which economic loss due to pests and the cost of pest control depend on the pest density, denoted $\theta$. The EIL is then given by the level of $\theta$ where the reduction of loss equals the cost of control. Terminology and symbology follows Pedigo et al. (1986) where possible. For a list of symbols used in the following sections, see the glossary included below.

Assuming economic loss depends linearly on the pest density, we have the loss function

$$
L_{p e}(\theta)= \begin{cases}V D^{\prime} \theta & \text { if } 0 \leq \theta \leq \frac{1}{D^{\prime}} \\ V & \text { if } \theta>\frac{1}{D^{\prime}}\end{cases}
$$

where

$V$ is the maximal value of crop per unit (e.g. value per hectar),

$D^{\prime}$ is the loss associated with an increase of one of the pest density, and

$\theta$ is the pest density per unit.

The loss function is constant for $\theta>1 / D^{\prime}$, as loss is bounded by the maximal crop value $V$.

Under the assumption that the cost of pest control $C_{p e}$ is a constant (i.e. cost does not depend on $\theta$ ) in the interval $[0, V]$, the benefit of pest control is the reduction in loss minus the cost of control, i.e. $L_{p e}(\theta)-L_{p e}\left(\theta^{\prime}\right)-C_{p e}$, where $\theta$ and $\theta^{\prime}$ are pest densities before and after control respectively, and $C_{p e}$ is the cost of control per unit crop. The EIL is the smallest $\theta$ such that the benefit is greater than zero. Setting $\theta^{\prime}$ to zero (i.e. assuming pest control removes the entire population) and solving for $\theta$ gives a basic expression for the EIL: 


$$
\begin{gathered}
L_{p e}(\theta)-L_{p e}(0)-C_{p e} \geq 0 \Longleftrightarrow \\
V D^{\prime} \theta \geq C_{p e} \Longleftrightarrow \\
\theta \geq \frac{C_{p e}}{V D^{\prime}} .
\end{gathered}
$$

Hence, pest control is economically beneficial if the pest density exceeds the EIL, which is given by the right-hand side of the final inequality.

This situation is illustrated in Fig. 2.1. The EIL is given by the projection of the intersection of the loss function and the cost of pest control. For pest densities below the EIL, the benefit of pest control is negative as the cost is higher than the current loss; for pest densities above the EIL, the loss exceeds the cost, so control measures are beneficial.

Before turning to the pollinator case, we look to relax the assumption that pest control is completely successful. Let $K_{p e}$ denote the success proportion of pest control, so that e.g. $K_{p e}=0.25$ would signify that pest control cuts the pest population by one fourth. The economic gain of pest control is then given by

$$
L_{p e}(\theta)-L_{p e}\left(\left(1-K_{p e}\right) \theta\right)-C_{p e},
$$

i.e. the reduction in loss due to pests as the pest density moves from the current level $\theta$ to $\left(1-K_{p e}\right) \theta$, minus the cost of control. The EIL when taking the success of control into account is then derived as

(a)

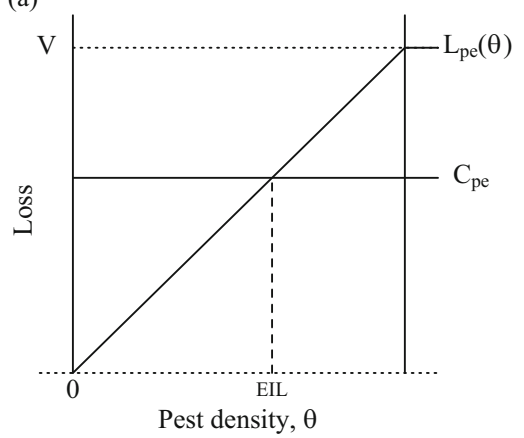

(b)

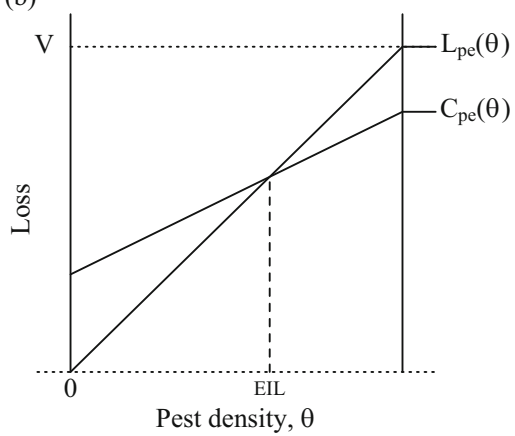

Fig. 2.1 Illustrations of the EIL for (a) the case of constant cost and (b) the case with pest densitydependent cost. The EIL is given by the projection of the intersection of the cost function, $C_{p e}$ or $C_{p e}(\theta)$, and the loss function $L_{p e}(\theta)$. For densities below the EIL, the cost of pest control is greater than the current loss due to pests, meaning that pest control is economically unsound. For densities above the EIL, the current loss is greater than cost of control, meaning that there is an economical gain from pest control. The illustrations are under the assumption that pest control reduces the pest density to zero 


$$
\begin{gathered}
L_{p e}(\theta)-L_{p e}\left(\left(1-K_{p e}\right) \theta\right)-C_{p e} \geq 0 \Longleftrightarrow \\
V D^{\prime} \theta-V D^{\prime}\left(1-K_{p e}\right) \theta-C_{p e} \geq 0 \Longleftrightarrow \\
\theta \geq \frac{C_{p e}}{V D^{\prime} K_{p e}} .
\end{gathered}
$$

This derived EIL is equivalent to adjusting the cost of pest control by the success rate. Note that this derivation assumes $\theta \leq 1 / D^{\prime}$.

\subsubsection{Pollinator Economic Impact Level with Constant Cost}

We turn now to the case of pollinator management and derive a measure of economic impact in analog to the economic injury level for pests. As in the pest case, the total value of one unit of crop is denoted $V$. Following Gallai and Salles (2016), a proportion $\bar{D}$ (the pollinator dependence) of $V$ is said to depend on pollinators, while the remaining part $(1-\bar{D}) V$ depends on other factors, e.g. self-pollination and pollination from insects other than the primary pollinator. We introduce an optimal density $\phi_{o p t}$, which is a level of pollinator density such that the marginal decrease in loss (i.e. pollinator limitation of yield) as the density increases is approximately zero. The maximal pollinator-dependent loss is $\bar{D} V$ and it occurs when the pollinator density (here denoted $\phi$ ) is 0 .

Assuming that loss depends linearly on the pollinator density $\phi$ now gives the loss function

$$
L_{p o}(\phi)= \begin{cases}\bar{D} V-\bar{D} V \frac{\phi}{\phi_{o p t}} & \text { if } 0 \leq \phi \leq \phi_{o p t} \\ 0 & \text { if } \phi>\phi_{o p t}\end{cases}
$$

When the pollinator density is zero, the loss equals the value of one unit of crop times the pollinator dependence. The loss decreases linearly as the density increases and reaches zero at the optimal density $\phi_{\text {opt }}$, after which there is no marginal effect of increased density.

As in the case for pest control we can introduce a cost of pollinator management $C_{p o}$ and construct a function for the benefit of management as the decrease in loss minus the cost: $L_{p o}(\phi)-L_{p o}\left(\phi^{\prime}\right)-C_{p o}$. Under the assumption that pollinator management moves the pollinator density to, or above, the optimal density, we can derive a basic pollinator economic impact level (PEIL):

$$
\begin{gathered}
L_{p o}(\phi)-L_{p o}\left(\phi_{o p t}\right)-C_{p o} \geq 0 \Longleftrightarrow \\
\bar{D} V-\bar{D} V \frac{\phi}{\phi_{o p t}}-C_{p o} \geq 0 \Longleftrightarrow
\end{gathered}
$$




$$
\phi \leq \phi_{o p t} \frac{\bar{D} V-C_{p o}}{\bar{D} V} .
$$

Hence, the derivation shows that pollinator management is beneficial for observed pollinator densities below the PEIL, which is given by $\phi_{o p t}\left(\bar{D} V-C_{p o}\right) /(\bar{D} V)$.

The basic pollinator EIL is illustrated in Fig. 2.2. The loss function is linear from $\bar{D} V$ at $\phi=0$ to 0 at $\phi=\phi_{o p t}$ and the cost is a constant at $C_{p o}$. The PEIL is the projection of the intersection between cost and loss onto the $\phi$ axis. For $\phi$ below the PEIL, the loss is greater than the cost, and pollinator management is therefore economically beneficial.

A pollinator success rate can be included in the calculation by setting $\phi^{\prime}$ to $\phi+$ $K_{p o}\left(\phi_{o p t}-\phi\right)$. The rationale is that pollinator management reduces the distance to the optimal level $\phi_{\text {opt }}$ by a proportion given by the success rate. Simplifying and solving for $\phi$ gives a pollinator EIL under consideration of $K_{p o}$ as

$$
\begin{gathered}
L_{p o}(\phi)-L_{p o}\left(\phi+K_{p o}\left(\phi_{o p t}-\phi\right)\right)-C_{p o} \geq 0 \Longleftrightarrow \\
\bar{D} V-\bar{D} V \frac{\phi}{\phi_{o p t}}-\left(\bar{D} V-\bar{D} V \frac{\phi+K_{p o}\left(\phi_{o p t}-\phi\right)}{\phi_{o p t}}\right)-C_{p o} \geq 0 \Longleftrightarrow \\
\frac{\bar{D} V}{\phi_{o p t}} K_{p o}\left(\phi_{o p t}-\phi\right)-C_{p o} \geq 0 \Longleftrightarrow \\
\phi \leq \phi_{o p t} \frac{\bar{D} V K_{p o}-C_{p o}}{\bar{D} V K_{p o}} .
\end{gathered}
$$

(a)

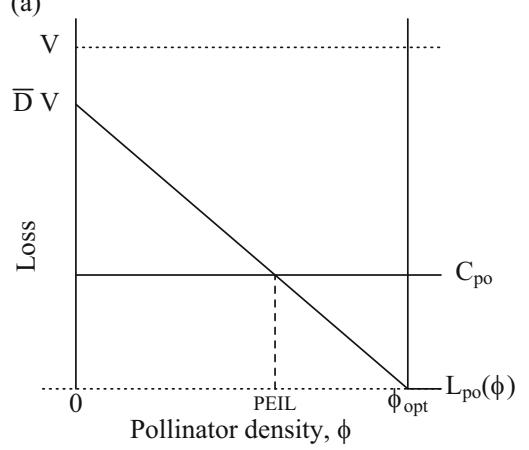

(b)

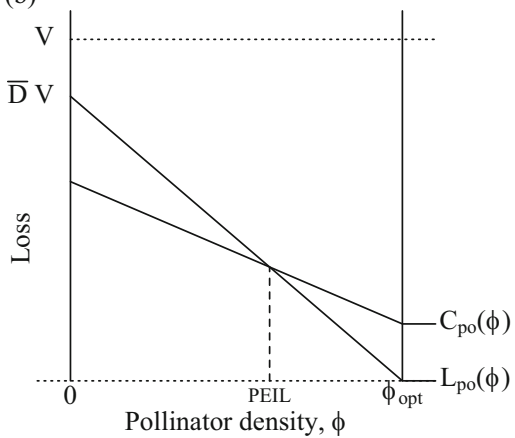

Fig. 2.2 Illustrations of the pollinator EIL for (a) the case of constant cost and (b) pollinator density-dependent cost. The PEIL is given by the projection of the intersection of the cost function, $C_{p o}$ or $C_{p o}(\phi)$, and the loss function $L_{p o}(\phi)$. For densities below the PEIL, the cost of pollinator management is greater than the loss due to a suboptimal pollinator density, while for densities above the PEIL, the loss is greater than cost, meaning there is an economical gain from pollinator management. The illustrations are under the assumption that pollinator management increases the pollinator density above the optimal pollinator density $\phi_{o p t}$ 
Hence, including the success rate $K_{p o}$ in the calculation gives a PEIL where the maximal value of the crop $V$ is adjusted by the success rate.

\subsubsection{Pest Economic Injury Level with Linear Cost}

We move now to the slightly more general situation where the cost of pest control depends linearly on the pest density. The cost function is given by

$$
C_{p e}(\theta)=C_{p e}+M_{p e} \theta,
$$

where $C_{p e}$ is a base cost and $M_{p e}$ is a marginal cost interpretable as the increased cost associated with an increase of one in pest density. If $M_{p e}$ is zero, the cost of pest control is constant and we return to the previously derived EIL for constant cost.

We set up a function for the benefit of control as the decrease in loss minus the cost:

$$
L_{p e}(\theta)-L_{p e}\left(\theta^{\prime}\right)-C_{p e}(\theta) .
$$

Given the assumptions that control gives a decrease in the pest density to $\left(1-K_{p e}\right) \theta$ and that cost is linear in $\theta$, the benefit function is

$$
V D^{\prime} \theta-V D^{\prime}\left(1-K_{p e}\right) \theta-C_{p e}-\theta M_{p e},
$$

where $V, D^{\prime}, K_{p e}$ have the same meaning as in the derivation of the EIL with constant cost. Setting the benefit of control greater than zero and solving for the pest density $\theta$ gives

$$
\begin{gathered}
V D^{\prime} \theta-V D^{\prime}\left(1-K_{p e}\right) \theta-C_{p e}-\theta M_{p e} \geq 0 \Longleftrightarrow \\
\theta \geq \frac{C_{p e}}{V D^{\prime} K_{p e}-M_{p e}},
\end{gathered}
$$

where the last term $C_{p e} /\left(V D^{\prime} K_{p e}-M_{p e}\right)$ is the EIL for linear cost. This derivation is done under the assumption that $\theta$ is less than $1 / D^{\prime}$. If $M_{p e}$ is zero, the EIL for linear cost reduces to the EIL for constant cost with a success rate $K_{p e}$.

The situation with density-dependent cost is illustrated in Fig. 2.1b. Both the loss function $L_{p e}(\theta)$ and the cost function $C_{p e}(\theta)$ increase with the pest density. The EIL is given by the value of $\theta$ where the lines intersect, as the benefit is less than zero below that EIL and greater than zero above. The point where the cost function cuts the y-axis is the base cost of pest control, i.e. $C_{p e}(0)=C_{p e}$.

Figure 2.1 also serves to illustrate two features of the model with densitydependent cost that are not present in the model with constant cost. Firstly, if the 
marginal cost is high in relation to the value $V$ and the damage associated with an increase in pest density $D^{\prime}$, it is possible that the loss function and the cost function do not intersect. In that case the benefit of pest control will be negative for all values of $\theta$ and the calculated EIL will be negative or a division by zero. Secondly, since the loss function $L_{p e}(\theta)$ is bounded by $V$, the loss function and the cost function can intersect twice. This can be visualized by extending the functions to higher values of $\theta$. Hence, the EIL has not only a lower limit to where pest control is beneficial, but also a higher limit.

\subsubsection{Pollinator Economic Impact Level with Linear Cost}

For the pollinator case, a cost function which depends linearly on the pollinator density $\phi$ can be defined as

$$
C_{p o}(\phi)=C_{p o}+\left(\phi_{o p t}-\phi\right) M_{p o} .
$$

Here, $C_{p o}$ is the baseline cost of pollinator management and $M_{p o}$ is the marginal increase of cost as the pollinator density decreases. For the calculations of the pollinator EIL, the baseline cost and the highest possible cost, attained at $\phi=0$, are both assumed to be less than the maximal value of the crop times pollinator dependence $(\bar{D} V)$.

As in previous cases, the PEIL is derived by forming a benefit function and solving for the pollinator density where the benefit of control is greater than zero. For the model with linear cost and a success rate $K_{p o}$ the benefit function is given by

$$
\begin{gathered}
L_{p o}(\phi)-L_{p o}\left(\phi+K_{p o}\left(\phi_{o p t}-\phi\right)\right)-C_{p o}(\phi)= \\
\bar{D} V-\bar{D} V \frac{\phi}{\phi_{o p t}}-\left(\bar{D} V-\bar{D} V \frac{\phi+K_{p o}\left(\phi_{o p t}-\phi\right)}{\phi_{o p t}}\right)-C_{p o}-\left(\phi_{o p t}-\phi\right) M_{p o} .
\end{gathered}
$$

Setting an inequality where the benefit is greater than zero and solving for $\phi$ gives the EIL of the model:

$$
\begin{gathered}
\bar{D} V-\bar{D} V \frac{\phi}{\phi_{o p t}}-\left(\bar{D} V-\bar{D} V \frac{\phi+K_{p o}\left(\phi_{o p t}-\phi\right)}{\phi_{o p t}}\right)-C_{p o}-\left(\phi_{o p t}-\phi\right) M_{p o}>0 \Longleftrightarrow \\
\phi<\phi_{o p t} \frac{\bar{D} V K_{p o}-C_{p o}-\phi_{o p t} M}{\bar{D} V K_{p o}-\phi_{o p t} M} .
\end{gathered}
$$

The calculation uses the assumption that $\phi$ is between 0 and $\phi_{\text {opt }}$. Note that the pollinator EIL of the model with linear cost equals the pollinator EIL (with success rate $K_{p o}$ ) for constant cost if $M_{p o}=0$. 
The situation with pollinator-dependent cost and $K_{p o}=1$ is illustrated in Fig. 2.2. Both cost and loss decrease with increasing pollinator density $\phi$. In the point $\phi=\phi_{\text {opt }}$ the loss is zero and the cost is equal to the baseline cost $C_{p o}$. The PEIL is given as the value of $\phi$ where the cost and the loss functions intersect, and pollinator management is beneficial for values of $\phi$ below the PEIL, i.e. the densities where the loss is greater than the cost.

\subsubsection{Non-linear Loss Functions}

The traditional EIL and the pollinator EIL presented here both rely on the assumption that economic loss depends linearly on pest or pollinator density. The justification of this assumption is twofold: the linear function is an approximation for most common functions used to model yield if one is interested in a small range of pest or pollinator density levels; and using linear loss results in simple expressions for the EIL. In this section we discuss some non-linear functions (variants of the sigmoid curve) which may be better approximations of the relation between insect density and yield. The discussion focuses on the case of pollinators.

We begin by looking at the connection between a function on yield and the loss function. Let $v(\phi)$ denote a measure of yield as a function of pollinator density. The sigmoid curve is given by

$$
v(\phi)=t_{1}+t_{2}\left(1-\exp \left\{-t_{3} \phi^{t_{4}}\right\}\right)
$$

Assuming $t_{3}$ and $t_{4}$ are both positive, the function is bounded from above by $t_{1}+t_{2}$. Hence, $1-v(\phi) /\left(t_{1}+t_{2}\right)$ gives a proportional loss at the pollinator density $\phi$, and multiplying that proportion by $V$ gives a loss function per unit crop. Furthermore, the loss at $\phi=0$ should equal $\bar{D} V$ (the value of the pollinator-dependent portion of the crop), the loss should decrease as $\phi$ increases, and be bounded from below by zero. These assumptions result in the following sigmoid loss function

$$
L_{p o}^{s}(\phi)=\bar{D} V \exp \left\{-t_{3} \phi^{t_{4}}\right\}
$$

The information loss of using a linear loss function depends on the in-going parameter values $\left(t_{3}\right.$ and $t_{4}$ for the sigmoid loss function, and $\phi_{o p t}$ for the linear loss function) and the range of densities taken into consideration. Figure 2.3 displays two examples of sigmoid loss functions and an approximated linear loss function. The parameter $\phi_{\text {opt }}$ is set to the smallest $\phi$ such that $L_{p o}^{s}(\phi)<\bar{D} V / 20$, i.e. the pollinator density where no more than $5 \%$ of the pollinator dependent crop value is lost. It is also possible to change the intercept at $\phi=0$ in order to get a better linear approximation of the sigmoid loss function. The figures illustrate the possible differences between the non-linear and the linear loss function, and how two different sigmoid loss functions can give rise to similar linear approximations. This highlights how any application of the EIL or pollinator EIL should take into 
(a)

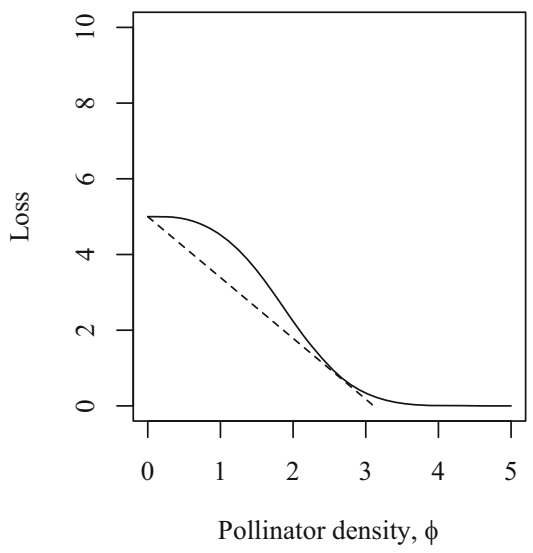

(b)

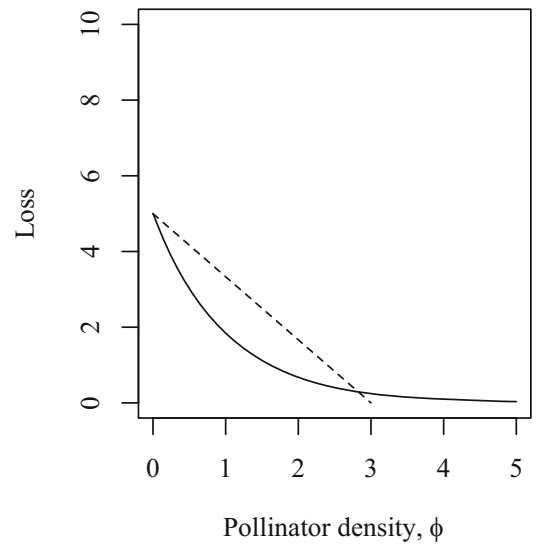

Fig. 2.3 Illustrations of non-linear $\left(L_{p o}^{s}(\phi)=\bar{D} V \exp \left\{-t_{3} \phi^{t_{4}}\right\}\right)$ and linear $\left(L_{p o}(\phi)=\bar{D} V-\right.$ $\left.\bar{D} V \phi / \phi_{\text {opt }}\right)$ loss functions for (a) parameters $\left(t_{3}, t_{4}\right)=(0.1,3)$ and $(\mathbf{b})$ parameters $\left(t_{3}, t_{4}\right)=$ $(1,1)$. $\phi_{\text {opt }}$ set to the density level where the non-linear curve equals $\bar{D} V / 20$. For (a) and (b), $V$ and $\bar{D}$ equals 10 and 0.5

consideration whether the use of a linear or non-linear curve serves as the most suitable description of relation between insect density and yield.

\subsubsection{A Joint EIL}

So far we have only looked at the univariate cases, where the EIL of the pest density has been derived without taking the pollinator density into account, and vice versa. We turn now to the problem of finding a beneficial action in a situation where both pest control and pollinator management are available, and both pest and pollinator density affect the total loss. The reasoning parallels the univariate cases: we set up loss and cost functions of the densities, examine the reduction in loss following a specific action, and identify levels where that action is economically beneficial.

Given the univariate loss functions $L_{p e}(\theta)$ and $L_{p o}(\phi)$, a natural joint loss function $^{1}$ is given by

\footnotetext{
${ }^{1}$ We justify this loss function by help of an example. Say that the value of one unit of crop is four, that the current pest density is associated with a loss of one third of the crop, and that the current pollinator density is associated with a loss of one sixth of the crop. After accounting for the loss due to the low pollinator density, five sixths of the total value remains. Of this, one third is lost due to the pest density, leaving two thirds of five sixths, or ten parts of eighteen. The total loss is thereby the lost portion, eight parts of eighteen, times four (the maximal value). This reasoning corresponds to the joint loss function $L(\theta, \phi)$.
} 


$$
L(\theta, \phi)=V\left(1-\left(1-\frac{L_{p e}(\theta)}{V}\right)\left(1-\frac{L_{p o}(\phi)}{V}\right)\right) .
$$

Note that the joint loss function reduces to a univariate case in the best case scenarios: $L(0, \phi)=L_{p o}(\phi)$ and $L\left(\theta, \phi_{o p t}\right)=L_{p e}(\theta)$. The joint loss function is in that sense an extension of the univariate cases. Inserting the expressions for the univariate loss functions $L_{p e}$ and $L_{p o}$ gives

$$
L(\theta, \phi)= \begin{cases}V\left(1-\left(1-D^{\prime} \theta\right)\left(1-\bar{D}+\bar{D} \frac{\phi}{\phi_{o p t}}\right)\right) & \text { if } \theta \leq \frac{1}{D^{\prime}} \text { and } \phi \leq \phi_{o p t} \\ V & \text { if } \theta>\frac{1}{D^{\prime}} \\ V D^{\prime} \theta & \text { if } \phi>\phi_{\text {opt }} \text { and } \theta<\frac{1}{D^{\prime}}\end{cases}
$$

The densities $\theta$ and $\phi$ are of course bounded from below by zero.

The joint loss function is illustrated in Fig. 2.4. The function is zero when the pest density $\theta$ is zero and the pollinator density $\phi$ is greater than the optimal level. For fixed $\phi$, the function is linear in $\theta$ and vice versa. The function is bounded by the total value $V$, which can be seen in the constant section where $\theta$ is large.

In the univariate cases we set up a function for the benefit of control as the reduction in loss, minus the cost. This is not as straightforward in the joint case since there are multiple possible strategies and since control measures of one kind can have dynamic effects, i.e. pest control can affect the pollinator density and pollinator management can affect the pest density. In the following section we assume that applying both control measures is effectively similar to pest control followed by pollinator management, that the effect of pest control on pollinator

(a)

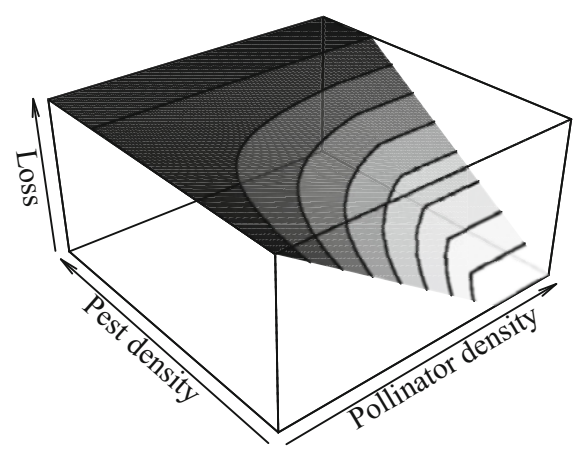

(b)

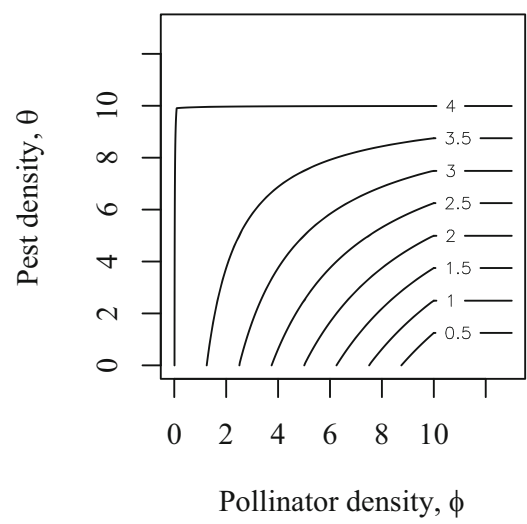

Fig. 2.4 Illustration of the joint loss function as a surface model and as contour lines for the model with parameters $\left(V, \bar{D}, D^{\prime}, \phi_{\text {opt }}\right)=(4,1,0.1,10)$. Loss has a negative relation to pollinator density and a positive relation to pest density 
density is a multiplicative factor $J_{p e}$, and that the effect of pollinator management on pest density is a multiplicative factor $J_{p o}$. For example, $J_{p e}=0.8$ would indicate a $20 \%$ decrease in the pollinator density after pest control, while $J_{p o}=1.1$ would indicate a $10 \%$ increase in the pest density after pollinator management.

In the joint model there are four available strategies: inaction $\left(S_{0}\right)$, pest control $\left(S_{p e}\right)$, pollinator management $\left(S_{p o}\right)$, and both $\left(S_{p e, p o}\right)$. The joint cost function is given by the unvariate cost function for strategies $S_{p e}$ and $S_{p o}$, and by the sum of the cost functions for strategy $S_{p e, p o}$. In the latter case, the assumption that pest control affects the pollinator density $\phi$ means that the cost of pollinator management is calculated based on the density $J_{p e} \phi$, i.e. the pollinator density after applying pest control. These assumptions allow us to formalize a joint cost function as a function of the pest density, the pollinator density, and the chosen strategy:

$$
C(\theta, \phi, S)= \begin{cases}0 & \text { if } S=S_{0} \\ C_{p e}+M_{p e} \theta & \text { if } S=S_{p e} \\ C_{p o}+\left(\phi_{o p t}-\phi\right) M_{p o} & \text { if } S=S_{p o} \\ C_{p e}+M_{p e} \theta+C_{p o}+\left(\phi_{o p t}-J_{p e} \phi\right) M_{p o} & \text { if } S=S_{p e, p o} .\end{cases}
$$

Note that the term $J_{p e} \phi$ can exceed $\phi_{o p t}$.

The benefit of control can now be calculated as the reduction in loss minus the cost of control. This gives a function of the pest and pollinator densities and the choice of action:

$$
L(\theta, \phi)-L\left(\theta^{\prime}, \phi^{\prime}\right)-C(\theta, \phi, S)
$$

The densities after control measures $\left(\theta^{\prime}\right.$ and $\left.\phi^{\prime}\right)$ depend on the type of control. In analog to the univariate cases, we assume that pest control and pollinator management have success rates $K_{p e}$ and $K_{p o}$ respectively. Under these assumptions, pest control decreases the pest density from $\theta$ to $\left(1-K_{p e}\right) \theta$ and changes the pollinator density from $\phi$ to $J_{p e} \phi$. Pollinator management increases the pollinator density from $\phi$ to $\phi+K_{p o}\left(\phi_{o p t}-\phi\right)$, while the pest density changes from $\theta$ to $J_{p o} \theta$. Pest control followed by pollinator management changes the pest density from $\theta$ to $J_{p o}(1-$ $\left.K_{p e}\right) \theta$ and changes the pollinator density from $\phi$ to $J_{p e} \phi+K_{p o}\left(\phi_{o p t}-J_{p e} \phi\right)$, which can be slightly simplified to $\left(1-K_{p o}\right) J_{p e} \phi+K_{p o} \phi_{o p t}$.

Putting the loss function, the cost function and the effects of control together gives the following expressions for the economic consequence of each possible action:

$$
\begin{cases}0 & \text { if } S=S_{0} \\ L(\theta, \phi)-L\left(\left(1-K_{p e}\right) \theta, J_{p e} \phi\right)-C\left(\theta, \phi, S_{p e}\right) & \text { if } S=S_{p e} \\ L(\theta, \phi)-L\left(J_{p o} \theta, \phi+K_{p o}\left(\phi_{o p t}-\phi\right)\right)-C\left(\theta, \phi, S_{p o}\right) & \text { if } S=S_{p o} \\ L(\theta, \phi)-L\left(J_{p o}\left(1-K_{p e}\right) \theta,\left(1-K_{p o}\right) J_{p e} \phi+K_{p o} \phi_{o p t}\right)-C\left(\theta, \phi, S_{p e, p o}\right) & \text { if } S=S_{p e, p o}\end{cases}
$$


In the univariate case we got a single expression for the economic benefit of control (since there was only one possible control measure), and an EIL could be calculated as the level where the benefit was greater than zero. In the joint case, all possible actions must be compared, and the straightforward calculation used in the univariate case is not possible.

Instead of calculating the analytical solution, we move on to present computational results. The computations are performed by setting up a grid of possible combinations of $\theta$ and $\phi$ for a specified set of ingoing parameter values, and calculating the economic consequence of each of the four strategies. Figure 2.5 illustrates the maximizing action for two different sets of ingoing parameter values.

Figure 2.5 (top) illustrates a symmetric situation where the marginal loss due to an increase in pest density equals the marginal loss due to a decrease in pollinator density, and the costs of pest control and pollinator management are equal. For cases where the pest density is low and the pollinator density high (the lower right corner of the plot), the most beneficial action is inaction. Pollinator management is the most beneficial action if both the pollinator and the pest densities are low (the lower left). Pest control is beneficial if both densities are high (top right). Both pest and pollinator management are the most beneficial action up to a point where the total cost of control measures exceed the value of the crop.

Figure 2.5 (bottom) illustrates the case where pest control is cheaper than pollinator management and less successful. The success proportion of pest control, $K_{p e}$, is set to 0.6 , i.e. pest control reduces the pest population by $60 \%$. We also assume pest control reduces the pollinator density by $20 \%\left(J_{p e}=0.8\right)$. The resulting graph of maximizing action shows an irregular pattern. Pest control is the maximizing action in the most extreme case of high pollinator density and high pest density, while pollinator management and a combination of pest control and pollinator management are most beneficial for cases of low pollinator density and low pest density.

\subsection{An Empirical Example}

We give an empirical example of a joint EIL for strawberry based on published data. Data on pests is taken from Mailloux and Bostanian (1988), a study on tarnished plant bug. Data on pollinators is from Bartomeus et al. (2014).

Mailloux and Bostanian (1988) estimate the mean standardized weight (the weight measure is a percent of the weight if there is no pest damage) of strawberry as a non-linear function of the pest density, measured as number of nymphs per blossom cluster. We present the basic model with some altered terminology, in order to be consistent with previous sections. Note especially the meaning of $V$, which in the article is the value in kilos, but here is the total value in ha. The predicted standardized weight is modelled by

$$
w(\theta)=\min \left(t_{1}+t_{2}\left(1-e^{t_{3} \theta}\right), 100\right),
$$




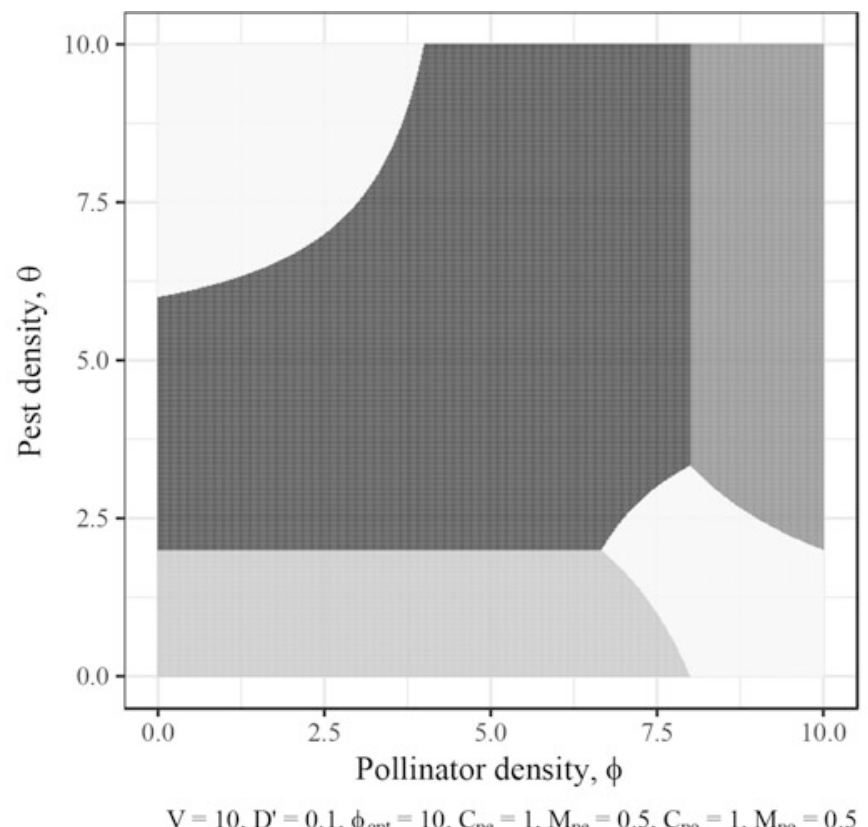

Maximizing action
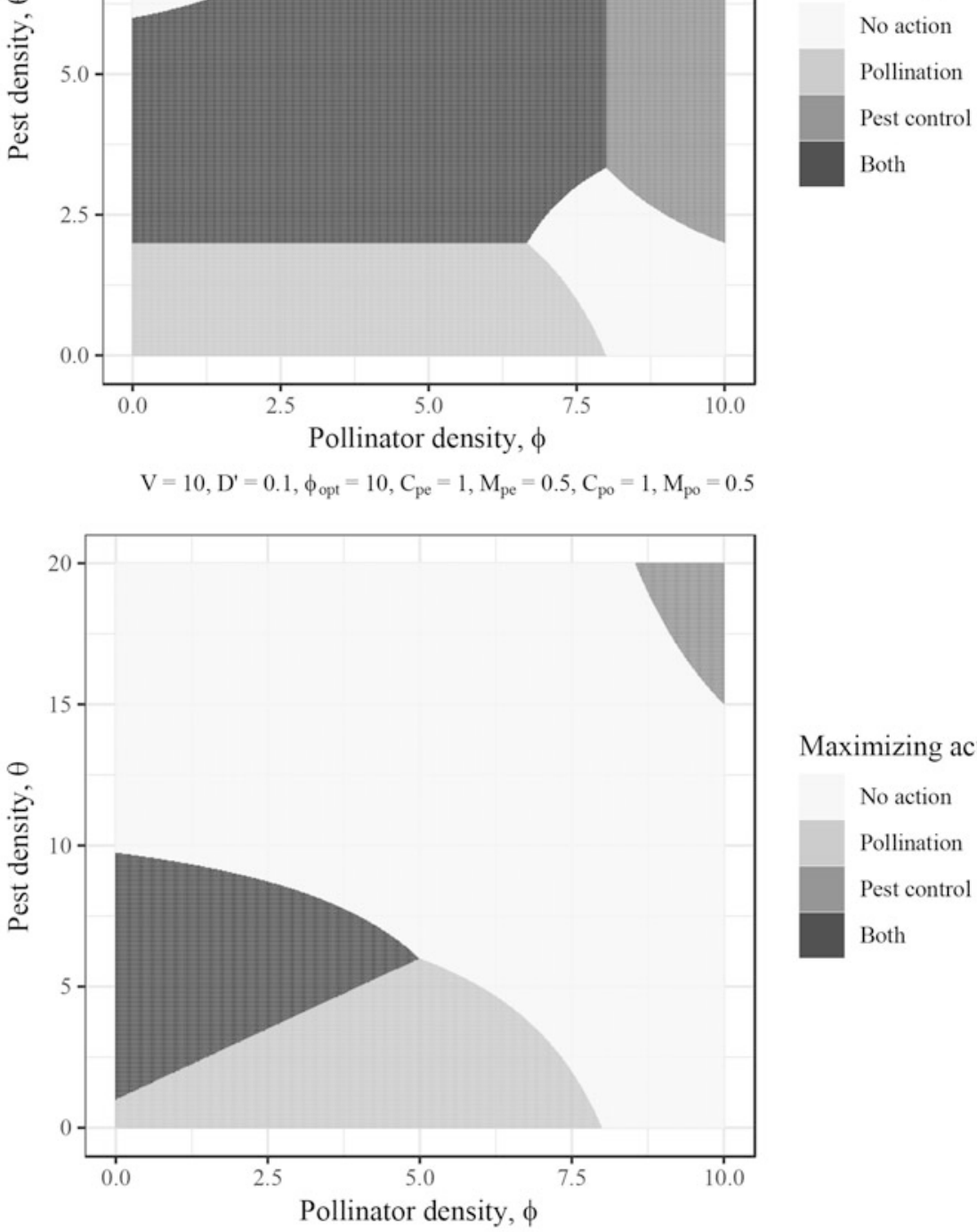

Maximizing action

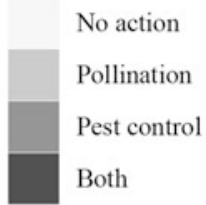

$\mathrm{V}=10, \mathrm{D}^{\prime}=0.05, \phi_{\text {opt }}=10, \mathrm{C}_{\mathrm{pe}}=0.1, \mathrm{M}_{\mathrm{pe}}=0.2, \mathrm{C}_{\mathrm{po}}=1, \mathrm{M}_{\mathrm{po}}=0.5, \mathrm{~K}_{\mathrm{pe}}=0.6, \mathrm{~J}_{\mathrm{pe}}=0.8$

Fig. 2.5 Illustration of the most economically beneficial action for combinations of pest and pollinator densities, for two different sets of parameters. Ingoing parameter values given in the graph. The top graph illustrates a symmetric situation where pest control and pollinator management are equally successful and beneficial. The bottom graph illustrates a situation where pest control has reduced success rate $\left(K_{p e}=0.6\right)$ and negative impact on the pollinator density $\left(J_{p e}=0.8\right)$ 
where $t_{1}, t_{2}$ and $t_{3}$ are parameters estimated from data and $\theta$ is the pest density. The function is a sigmoid curve with $t_{4}=1$, bounded by 100 . We will use the results pooled for the L'Assomption test site, for which the values of $t_{1}, t_{2}$ and $t_{3}$ are $110.649,-72.789$ and -0.207 . Given those parameter values, the weight is greater than zero for all possible values of $\theta$.

The cost-benefit relation is formalized as

$$
C_{p e}=K_{p e} V \frac{100-w(\theta)}{100},
$$

where the cost of control $C_{p e}$ is set to 100 , the success rate $K_{p e}$ is 0.9 (or $90 \%$ ), and the total value $V$ is 10,000 . The right-hand side of the the cost-benefit relation corresponds to what we have previously denoted $L_{p e}(\theta)$, the economic loss due to pests at the pest density level $\theta$. Inserting the weight function into the cost-benefit relation and solving for the pest density $\theta$ gives an EIL of 0.85 . In the original article an additional argument based on plant carrying capacity is used, giving a final EIL of 0.99. We will use the calculated value from the parametrized curve (i.e. 0.85), mainly for the direct comparison with the results for the joint EIL.

These results are visualized in Fig. 2.6. The loss function $L_{p e}(\theta)$ is the right-hand side of the cost-benefit relation and the horizontal line is the cost of control $C_{p e}$. The EIL is the projection of the intersection of the two curves.

Moving on, we look at the data of Bartomeus et al. (2014) and calculate a pollinator EIL. Here, the relation between mean berry weight and pollinator abundance (measured as total number of visits in a set time span) is given as a linear function. In order to calculate an EIL, we make the additional assumption that the goal mean berry weight is 15 grams. This results in a weight function given by

(a)

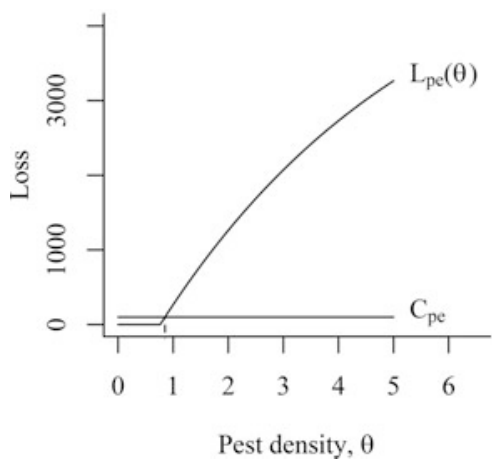

(b)

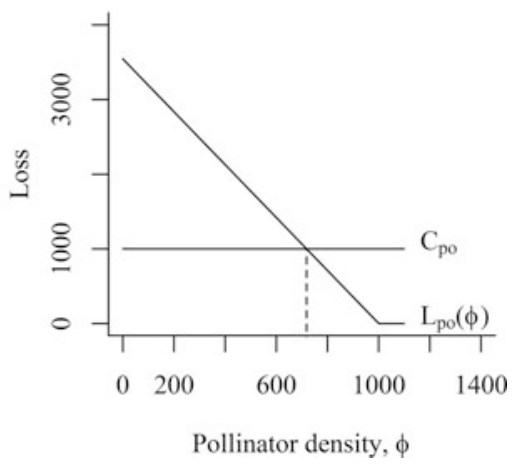

Fig. 2.6 Illustrations of the EIL and PEIL of the empirical examples. The loss function due to (a) pests and cost of pest control and the loss function due to (b) non-optimal pollination and cost of pollinator management. The EIL and pollinator EIL are given by the projection of the intersection of the loss function and the cost function 


$$
v(\phi)=\min \left(\beta_{0}+\beta_{1} \phi, 15\right),
$$

where $\beta_{0}$ and $\beta_{1}$ are parameters estimated from the data. Ordinary least squares estimation on the data for strawberry presented in the article gives the estimates $\beta_{0}=9.6827$ and $\beta_{1}=0.0053$.

We set up a cost-benefit relation as

$$
C_{p o}=V-V \frac{v(\phi)}{15} .
$$

As in the pest example, the right-hand side corresponds to the loss function, $L_{p o}(\phi)$. The value $V$ is set to 10,000 , taken from Mailloux and Bostanian (1988), and the cost of pollinator management $C_{p o}$ is set to 1000 . Given these parameters, a pollinator EIL is calculated by solving the cost-benefit relation for $\phi$, resulting in a value of 718.35 .

Since the loss function is linear, it is possible to translate the empirical loss function given in terms of predicted weight to the loss function presented in the derivation of the pollinator EIL. If the pollinator dependence is given by $\bar{D}=$ $1-\beta_{0} / 15$ and the optimal pollinator density by $\phi_{o p t}=15 \cdot \bar{D} / \beta_{1}$, the loss function can be re-written

$$
L_{p o}(\phi)=V-V \frac{\beta_{0}+\beta_{1} \phi}{15}=\bar{D} V-\bar{D} V \frac{\phi}{\phi_{o p t}},
$$

i.e. the same formulation of the loss function we have seen previously.

The pollinator EIL is illustrated in Fig. 2.6. The loss function decreases for $\phi$ less than about 1000 . For greater $\phi$ the optimal mean berry weight of 15 is reached and the estimated loss is zero. The cost is a constant at the value of $C_{p o}$. The pollinator EIL is given as the projection of the intersection between the cost and loss functions.

We can now set up a joint EIL by defining the joint loss function

$$
L(\theta, \phi)=V\left(1-\left(1-\frac{L_{p e}(\theta)}{V}\right)\left(1-\frac{L_{p o}(\phi)}{V}\right)\right),
$$

where $V$ is $10,000, L_{p e}(\theta)$ is the right-hand side of the cost-benefit relation for pest, and $L_{p o}(\phi)$ is the right-hand side of the cost-benefit relation for pollinators. Based on this joint loss function, the reduced loss and cost of each of the four strategies (no action, pest control, pollinator management, and both) are calculated and compared. We assume pest control and pollinator management are completely effective in the sense that pest control brings the pest density to zero and that pollinator management brings the pollinator density above the optimal level (which is about 1000), and that there is no effect of pest control on the pollinator density or of pollinator management on the pest density.

The results of applying the joint EIL to the empirical data is given in Fig. 2.7 (top). The black lines signify the EIL and pollinator EIL for the univariate cases. 


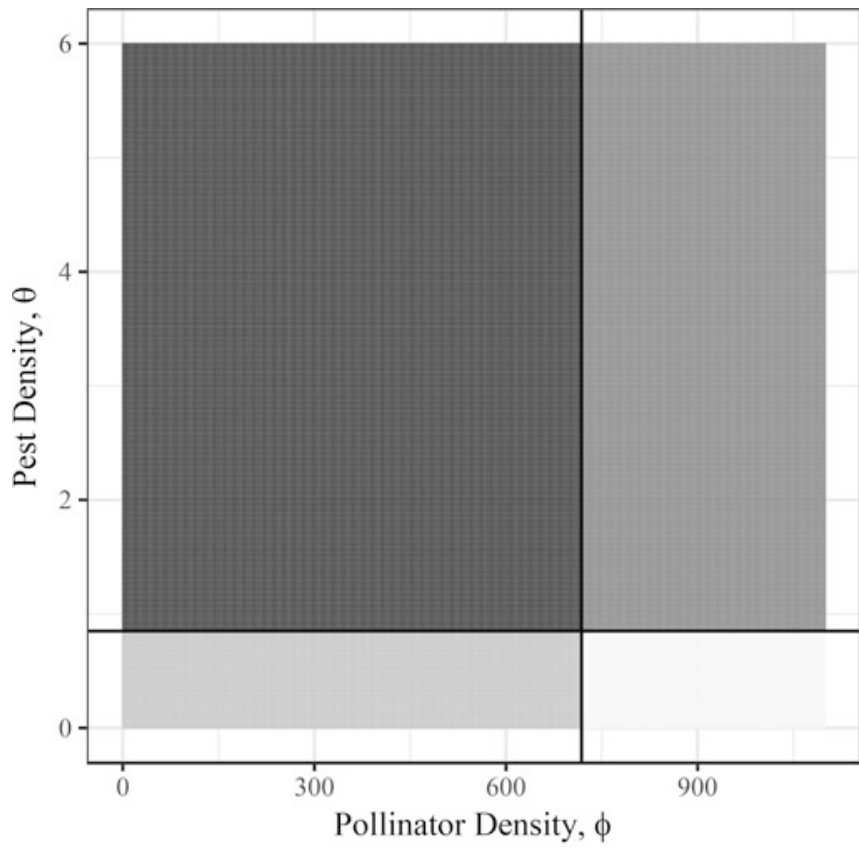

Maximizing action

No action

Pollination

Pest control

Both

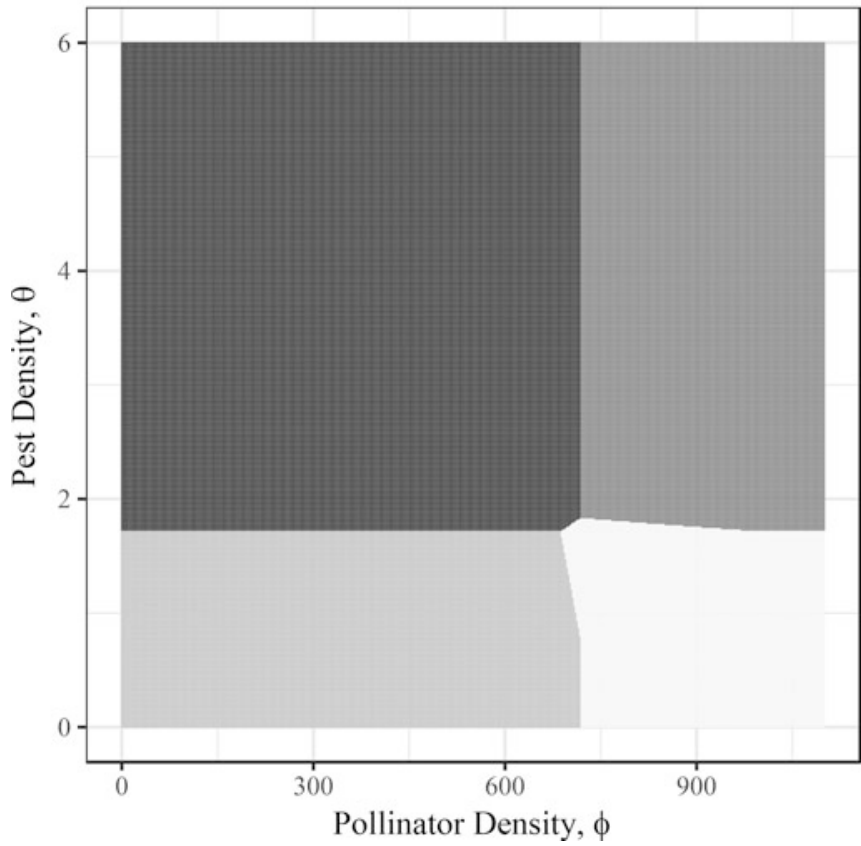

Maximizing action

No action

Pollination

Pest control

Both

Fig. 2.7 Illustration of the most economically beneficial action for combinations of pest and pollinator densities for the strawberry empirical data. Cost of pest control is set to (top) 100 and (bottom) 1000. In the case of a low cost of pest control, the areas connected to each strategy are rectangular, indicating that the most beneficial decision according to the joint EIL is similar to applying the EIL and the pollinator EIL separately. In the case of a higher cost of control, the areas become more irregular and the suggested strategy according to the joint EIL may differ from the EIL or pollinator EIL 
The four sections for the possible actions are almost completely rectangular, which indicates very small differences between applying the joint EIL and applying the EIL and the pollinator EIL independently. This is due to particular features in the loss functions, in particular the constant sections in the loss functions (see Fig. 2.6) and the low cost of pest control in relation to crop value. Figure 2.7 (bottom) shows the results of applying the joint EIL for the case where the cost of pest control is set to 1000 . The increased cost of pest control leads to a higher EIL, which is reflected in the decreased area connected to pest control. Increasing the cost also reveals that the regions are not complectely rectangular, meaning that there is some difference between applying the joint EIL and applying the univariate EILs seperately.

\subsection{Discussion}

\subsubsection{Flexibility of Use}

The joint EIL can facilitate IPPM decision making in recommending which action (i.e. management of pest, pollinators, both, or neither) represents the most economically optimal solution, under the management practices considered. Key to this functionality is its broad flexibility, and in particular the possibility to parameterize the joint EIL in a variety of different ways, depending on the scenario at hand, and the information available. Furthermore, this decision metric can be adapted for use for any pest type and control strategy, regardless of whether interactions (interferences or synergisms) with or from pollinators are apparent.

Flexibility in how to treat the cost of management in calculating jEILs offers one particular advantage. Cost of management may be either fixed (density independent) or linearly related to the scale of intervention (density dependant). Fixed costs can hence better represent certain management practices (e.g. use of high-efficiency chemical pesticides), where a fixed level of intervention is usually effective at alleviating a problem (e.g. high or low pest densities) regardless of scale (Pimentel and Levitan 1986). In contrast, linear costs often typify other pest and pollinator management practices, such as the use of commercial biocontrol agents and pollinators, or the creation of flower strips (Garibaldi et al. 2016).

A further advantage to the joint EIL is its ability to accommodate a variety of curve types describing pest and pollinator yield-density relationships. Core dependency on this parameter also represents a potential challenge, however, in that such empirical relationships (at least for pollinators) are not widely known outside of the major crops. Lack of such information does not necessarily preclude the use of joint EILs however, where it is possible to reasonably approximate missing parameters. For instance, where the pollinator loss function is unknown for a given crop, evidence supports that a linear (as opposed to non-linear) relationship could in theory serve as a reasonable approximation (Morris et al. 2010). Likewise, the recommended stocking density of managed pollinators, available for many 
crops (Breeze et al. 2014; Delaplane et al. 2000), could in many cases serve to reasonably estimate optimal pollinator density. In addition, the point at which the yield-density curve intercepts the Loss axis (as in Figs. 2.2 and 2.3) describes the pollinator-dependency of the crop. Qualitative approximations of this parameter appear possible for a majority of pollinator-dependant crops (see Klein et al. 2007).

\subsubsection{Dynamics}

Similar to the traditional EIL, the joint EIL is best viewed as a dynamic metric; in that as input parameters change across the phenological development of a crop, so too will the calculated joint EIL. For instance, pest control measures are likely to be prioritised by the joint EIL in the early crop development stages, due to the detrimental impact of pest injury at this time, and the lower potential for pest control measures to impact pollinators outside of the flowering period. In such scenarios, the joint EIL will closely (if not precisely) equate to the EIL, and no disadvantage is incurred from its use when only pests are of concern. In contrast, as a crop approaches flowering, the joint EIL can increasingly weigh the potential need for pollinator management, as well as any interactive effects on - or from - pest control.

In order to precisely ascertain the benefit of pollinator management, careful consideration must also be afforded to setting the optimal pollinator level (i.e. the target density towards which management strives). Possibilities include whether to fix this level as a season-wide average, based on the general pollination needs of the crop, or if this level should more dynamically track the crop's pollination needs across the flowering season - e.g. as governed by changes in flower density.

\subsubsection{Action Thresholds}

In order to make the joint EIL applicable, Action Thresholds (ATs) are required for both pests $\left(A T_{p e}\right)$ and pollinators $\left(A T_{p o}\right)$. Such ATs function much the same as the traditional AT, in that they define a density that should trigger an intervention in order to prevent the joint EIL being reached. Setting ATs for the joint EIL can be achieved by fixing $A T_{p e}$ and $A T_{p o}$ within the no action margin of the jEIL analytical plot (e.g. Fig. 2.5). Traditionally, the AT is set relatively far away (20-80\%) from the EIL - especially if there is a risk that a pest may vector even more harmful phytopathogens. The distance of the pest and pollinator AT from the three possible actions (pest control, pollinator management, or both) may hence differ; depending, for instance, on the lag time between a specific intervention and materialization of its effect. 


\subsubsection{Application in Practice}

In practice, the joint EIL can be used by agricultural advisors, farmers, and other stakeholders as a tool to support decision making, or simply to weigh up or explore (using real, simulated, or projected data) the implications of specific scenarios or management strategies. However, crop-specific knowledge is no doubt fundamental to the parameterization of the joint EIL; a task which may be best fulfilled by agricultural advisors or other specialists. Similar to the EIL, calculation of the joint EIL relies heavily upon several economic parameters (e.g. the current market value of yield, input costs, etc.). Economic information of this sort is likely to fluctuate regularly, and hence must be kept up-to-date in order to best inform decision making.

Once basic parameterization of jEIL is complete for a crop, monitoring and input of pest and pollinator field densities are the only further requirement for its use. This step can be managed either by advisors or farmers directly. Regular monitoring of pest densities is an established routine in IPM (Pedigo et al. 1986). However, for pollinators, field monitoring (e.g. through focal observations or active or passive sampling) is relatively less common for the purpose of informing decision making. Rather, management still typically hinges on the calculation of managed bee stocking rates (Delaplane et al. 2000). Use of the joint EIL, as part of an adaptive management approach, in this sense calls for more active practices of pollinator monitoring.

The arithmetic complexity of the joint EIL without doubt poses a challenge to its practical use. To address this issue, a simple graphical interface for the calculation of jEILs has been developed (presently in beta version). This interface will provide users with both graphical-based input options in addition to quantitative and graphical outputs (such as depicted in Figs. 2.5 and 2.7). We hope that this interface, together with future conceptual development of the joint EIL, can stimulate uptake of this decision metric among the scientific and agricultural community.

\section{Used Symbols}

\begin{tabular}{l|l}
\hline Symbol & \\
\hline$\theta$ & The pest density \\
\hline$L_{p e}(\theta)$ & Economic loss as a function of pest density \\
\hline$V$ & Value of one crop unit \\
\hline$D^{\prime}$ & Economic damage associated with on unit pest density \\
\hline$C_{p e}$ & Base cost of pest control \\
\hline$\theta^{\prime}$ & Pest density after pest control \\
\hline$K_{p e}$ & Success rate of pest control \\
\hline $\bar{D}$ & $\begin{array}{l}\text { The proportion of the crop dependent on pollinators (pollinator } \\
\text { dependence) }\end{array}$ \\
\hline
\end{tabular}




\begin{tabular}{l|l}
\hline$\phi$ & The pollinator density \\
\hline$\phi_{o p t}$ & The optimal pollinator density \\
\hline$L_{p o}(\phi)$ & Economic loss as a function of pollinator density \\
\hline$C_{p o}$ & Base cost of pollinator management \\
\hline$\phi^{\prime}$ & Pollinator density after pollinator management \\
\hline$K_{p o}$ & Success rate of pollinator management \\
\hline$M_{p e}$ & Marginal cost of pest control \\
\hline$M_{p o}$ & Marginal cost of pollinator management \\
\hline$v(\phi)$ & Yield as a function of pollinator density \\
\hline$L_{p o}^{s}(\phi)$ & Economic loss as a sigmoid function of pollinator density \\
\hline$L(\theta, \phi)$ & Economic loss as a joint function of pest and pollinator densities \\
\hline$J_{p e}$ & The effect of pest control on pollinator density \\
\hline$J_{p o}$ & The effect of pollinator management on pest density \\
\hline$S_{0}$ & The no action strategy \\
\hline$S_{p e}$ & The pest control strategy \\
\hline$S_{p o}$ & The pollinator management strategy \\
\hline$S_{p e, p o}$ & The pest control and pollinator management strategy \\
\hline$C(\theta, \phi, S)$ & $\begin{array}{l}\text { Cost of control as a function of pest and pollinator densities, and } \\
\text { strategy }\end{array}$ \\
\hline$A T_{p e}$ & Action threshold for pests \\
\hline$A T_{p o}$ & Action threshold for pollinators \\
\hline &
\end{tabular}

Acknowledgments The authors wish to thank Jan-Eric Englund for helpful input in developing the mathematical concepts here described. This work was funded by the Swedish Research Council Formas, grant no. 2017-02170 (to PAE), and grant no. 2016-00223 (to JAS).

\section{References}

Bartomeus I, Dicks L (2018) The need for coordinated transdisciplinary research infrastructures for pollinator conservation and crop pollination resilience. PeerJ Preprints 6:e26898v1

Bartomeus I, Potts SG, Steffan-Dewenter I, Vaissiere BE, Woyciechowski M, Krewenka KM, Tscheulin T, Roberts SPM, Szentgyorgyi H, Westphal C, Bommarco R (2014) Contribution of insect pollinators to crop yield and quality varies with agricultural intensification. PeerJ 2(1). ISSN: 2167-8359

Biddinger DJ, Rajotte EG (2015) Integrated pest and pollinator management-adding a new dimension to an accepted paradigm. Curr Opin Insect Sci 10:204-209

Breeze TD, Vaissière BE, Bommarco R, Petanidou T, Seraphides N, Kozák L, Scheper J, Biesmeijer JC, Kleijn D, Gyldenkærne S et al (2014) Agricultural policies exacerbate honeybee pollination service supply-demand mismatches across Europe. PLoS One 9(1):e82996

Brittain C, Potts SG (2011) The potential impacts of insecticides on the life-history traits of bees and the consequences for pollination. Basic Appl Ecol 12(4):321-331

Delaplane KS, Mayer DR, Mayer DF (2000) Crop pollination by bees. CABI, Wallingford

Dicks LV, Viana B, Bommarco R, Brosi B, del Coro Arizmendi M, Cunningham SA, Galetto L, Hill R, Lopes AV, Pires C et al (2016) Ten policies for pollinators. Science 354(6315):975-976

Egan PA, Dicks LV, Hokkanen HMT, Stenberg JA (2020) Delivering integrated pest and pollinator management (IPPM). Trends Plant Sci, in press 
Gallai N, Salles J-M (2016) Adaptation of an economy facing pollinator decline: a prospective analysis from the French case. UN Food and Agriculture Organization with Routledge, pp 43 56

Garibaldi LA, Aizen MA, Cunningham SA, Harder LD, Klein A-M (2016) Incremental contribution of pollination and other ecosystem services to agricultural productivity: effects of service quantity and quality. UN Food and Agriculture Organization with Routledge, pp 33-42

Garibaldi LA, Requier F, Rollin O, Andersson GKS (2017) Towards an integrated species and habitat management of crop pollination. Curr Opin Insect Sci 21:105-114

Godfray HCJ, Blacquiere T, Field LM, Hails RS, Petrokofsky G, Potts SG, Raine NE, Vanbergen AJ, McLean AR (2014) A restatement of the natural science evidence base concerning neonicotinoid insecticides and insect pollinators. Proc R Soc Lond B Biol Sci 281(1786):20140558

Isaacs R, Williams N, Ellis J, Pitts-Singer TL, Bommarco R, Vaughan M (2017) Integrated crop pollination: combining strategies to ensure stable and sustainable yields of pollinationdependent crops. Basic Appl Ecol 22:44-60

Klein A-M, Vaissiere BE, Cane JH, Steffan-Dewenter I, Cunningham SA, Kremen C, Tscharntke T (2007) Importance of pollinators in changing landscapes for world crops. Proc R Soc Lond B Biol Sci 274(1608):303-313

Mailloux G, Bostanian J (1988) Economic injury level model for Tarnished Plant Bug, Lygus lineolaris (Palisot de Beauvois) (Hemiptera: Miridae), in strawberry fields. Environ Entomol 17(3):581-586

Morris WF, Vázquez DP, Chacoff NP (2010) Benefit and cost curves for typical pollination mutualisms. Ecology 91(5):1276-1285

Oerke E-C (2006) Crop losses to pests. J Agric Sci 144(1):31-43

Pedigo LP, Rice ME (2014) Entomology and pest management. Waveland Press, Long Grove

Pedigo LP, Hutchins SH, Higley LG (1986) Economic injury levels in theory and practice. Annu Rev Entomol 31(1):341-368

Pimentel D, Levitan L (1986) Pesticides: amounts applied and amounts reaching pests. Bioscience 36(2):86-91

Siviter H, Koricheva J, Brown MJF, Leadbeater E (2018) Quantifying the impact of pesticides on learning and memory in bees. J Appl Ecol 55(6):2812-2821

Stanley DA, Garratt MPD, Wickens JB, Wickens VJ, Potts SG, Raine NE (2015) Neonicotinoid pesticide exposure impairs crop pollination services provided by bumblebees. Nature 528(7583):548

Stenberg JA (2017) A conceptual framework for integrated pest management. Trends Plant Sci 22(9):759-769

Stern VM, Smith RF, van den Bosch R, Hagen K et al (1959) The integration of chemical and biological control of the spotted alfalfa aphid: the integrated control concept. Hilgardia 29(2):81-101

Open Access This chapter is distributed under the terms of the Creative Commons Attribution 4.0 International License (http://creativecommons.org/licenses/by/4.0/), which permits use, duplication, adaptation, distribution and reproduction in any medium or format, as long as you give appropriate credit to the original author(s) and the source, a link is provided to the Creative Commons licence and any changes made are indicated.

The images or other third party material in this chapter are included in the work's Creative Commons licence, unless indicated otherwise in the credit line; If such material is not included in the work's Creative Commons licence and the respective action is not permitted by statutory regulation, users will need to obtain permission from the license holder to duplicate, adapt or reproduce the material. 